\title{
Optical performance and radiation stability of polymer X-ray refractive nano-lenses
}

\begin{abstract}
Alexander Barannikov, Maxim Polikarpov, Petr Ershov, Vladimir Bessonov, Ksenia Abrashitova, Irina Snigireva, Vyacheslav Yunkin, Gleb Bourenkov, Thomas Schneider, Andrey A. Fedyanin and Anatoly Snigirev
\end{abstract}

J. Synchrotron Rad. (2019). 26, 714-719

\section{IUCr Journals CRYSTALLOGRAPHY JOURNALS ONLINE}

Copyright (C) International Union of Crystallography

Author(s) of this article may load this reprint on their own web site or institutional repository provided that this cover page is retained. Republication of this article or its storage in electronic databases other than as specified above is not permitted without prior permission in writing from the IUCr.

For further information see https://journals.iucr.org/services/authorrights.html 
JOURNAL OF SYNCHROTRON RADIATION

ISSN 1600-5775

Received 26 October 2018

Accepted 29 January 2019

Edited by A. Momose, Tohoku University, Japan

Keywords: full-field X-ray microscopy; X-ray refractive lenses; additive manufacturing.

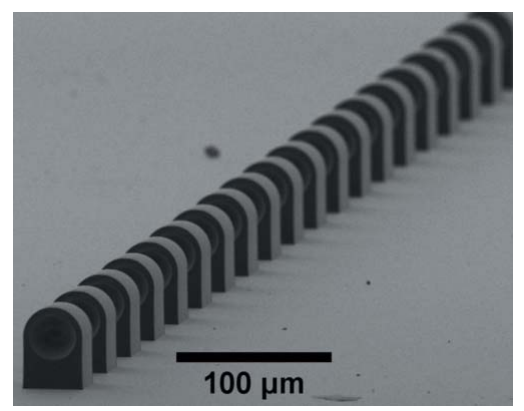

C 2019 International Union of Crystallography

\section{Optical performance and radiation stability of polymer X-ray refractive nano-lenses}

\author{
Alexander Barannikov, ${ }^{a}$ Maxim Polikarpov, ${ }^{\text {b* }}$ Petr Ershov, ${ }^{a}$ Vladimir Bessonov, ${ }^{\mathrm{c}, \mathrm{d}}$ \\ Ksenia Abrashitova, ${ }^{\mathrm{a}, \mathrm{c}}$ Irina Snigireva, ${ }^{\mathrm{e}}$ Vyacheslav Yunkin, ${ }^{\mathrm{f}}$ Gleb Bourenkov, ${ }^{\mathrm{b}}$ \\ Thomas Schneider, ${ }^{b}$ Andrey A. Fedyanin ${ }^{\mathrm{c}}$ and Anatoly Snigirev ${ }^{\mathrm{a}}$
}

\begin{abstract}
ammanuel Kant Baltic Federal University, Nevskogo 14, Kaliningrad 236041, Russia, ${ }^{\mathbf{b}}$ European Molecular Biology Laboratory, Hamburg Unit, Notkestraße 85, Hamburg, Germany, ${ }^{\mathbf{C}}$ Faculty of Physics, Lomonosov Moscow State University, Moscow 119991, Russia, ${ }^{\mathbf{d}}$ Frumkin Institute of Physical Chemistry and Electrochemistry, Russian Academy of Sciences, Moscow 119071, Russia, ${ }^{\mathbf{E} E u r o p e a n}$ Synchrotron Radiation Facility (ESRF), 71 avenue des Martyrs, 38043 Grenoble, France, and $\mathbf{f}^{\prime}$ Institute of Microelectronics Technology RAS, Chernogolovka, Russia.

*Correspondence e-mail: mpolikarpov@embl-hamburg.de
\end{abstract}

Full-field X-ray imaging and microscopy with polymer compound refractive nano-lenses is demonstrated. Experiments were carried out at beamline ID13 at the European Synchrotron and yielded a resolution of $100 \mathrm{~nm}$. The lenses were demonstrated to be functioning even after an absorbed dose of $\sim 10^{7} \mathrm{~Gy}$. This article also discusses issues related to lens aberrations, astigmatism and radiation stability, and thus ways of improving the lens further are considered. Polymer nano-lenses are versatile and are promissing for nano-focusing and compact $\mathrm{X}$-ray microscopy.

\section{Introduction}

In recent years, a new (fourth) generation of light sources has been emerging worldwide. Diffraction-limited machines with increased brilliance, coherence and flux of the X-ray beam are opening up ways to various promising experimental techniques. However, it also presents novel challenges for X-ray optics (Dimper et al., 2014) which should preserve unique radiation properties and be capable of beam transport, nanofocusing, phase-contrast imaging and full-field microscopy. The last issue becomes a complex task as highly coherent $\mathrm{X}$-ray radiation easily interacts on its path with various imperfections of optical elements, spoiling the outgoing wavefront with aberrations.

X-ray refractive lenses (Snigirev et al., 1996) are used as standard beamline optical elements (Lengeler et al., 1999, 2005; Aristov et al., 2000; Snigirev et al., 2007; Polikarpov et al., 2016) at many third-generation synchrotrons. Nowadays, beryllium and silicon lenses are the most commonly used owing to their well developed manufacturing process. Nevertheless, both types of lenses have their drawbacks.

Rotationally parabolic beryllium lenses are made of a polycrystalline metal with grain sizes of 10-100 $\mu \mathrm{m}$. Hence, the grain structure introduces parasitic phase contrast to the propagating wavefront, reducing the overall image quality in the full-field microscopic regime. In the context of nanofocusing applications, the diffraction-limited lens resolution is inversely proportional to the numerical aperture, which is the ratio of the lens aperture divided by its focal distance. The focal distance decreases with a decrease in the lens radius which allows one to achieve a higher resolution when approaching the diffraction limit. However, currently it is 
technologically difficult to produce beryllium lenses with a radius smaller than $50 \mu \mathrm{m}$, which in its turn limits the potentially achievable lens resolution to $50 \mathrm{~nm}$, while only $100 \mathrm{~nm}$ has been yet experimentally demonstrated (Snigireva et al., 2011; Lengeler, 2004; Snigirev \& Snigireva, 2008).

In contrast, parabolic silicon lenses are manufactured using the Bosch process (Aristov et al., 2001). They have ultra-small radii of about $5 \mu \mathrm{m}$ and are capable of focusing down to $50 \mathrm{~nm}$ (Schroer et al., 2005). Made of monocrystalline material, silicon lenses exhibit high structural perfection and are transparent to X-rays, thereby not introducing any parasitic phase contrast. However, as a consequence of the manufacturing by lithographic and etching processes, silicon lenses can only be structured along one dimension. This strongly limits their versatility and thus potential applicability for microscopy. When one needs two-dimensional focusing [e.g. for ptychographic or coherent diffraction imaging applications (Marchesini et al., 2003)], silicon lenses require complex crossalignment.

For the aforementioned applications, polymer refractive lenses were recently demonstrated to be efficient (Petrov et al., 2017; Sanli et al., 2018; Mirzaeimohri et al., 2018). Produced from a homogeneous polymer material by additive manufacturing, polymer lenses have compact dimensions, small radii and two-dimensional parabolic shapes. Thus, they can potentially be used for both uniform nano-focusing and shortfocus high-resolution full-field microscopy. In the present article, we investigate their focusing and imaging performance on third-generation synchrotrons. We also evaluate their radiation stability.

\section{Materials and methods}

The experiments presented in this paper were carried out at the ID13 beamline of The European Synchrotron, where an in-vacuum undulator produces $\mathrm{X}$-ray radiation $30 \mu \mathrm{m} \times$ $150 \mu \mathrm{m}$ in the vertical and horizontal directions at the source point, respectively. Then, X-rays pass through the cryogenically cooled $\mathrm{Si}(111)$ channel-cut monochromator to the experimental hutch, located $90 \mathrm{~m}$ from the source. We used $12.7 \mathrm{keV}$ photons in all experimental modes of this article.

In some parts of the experimental procedure, we used precollimation with a white-beam compound refractive lens (CRL), consisting of three beryllium double-concave parabolic refractive lenses with radius $R$ of $200 \mu \mathrm{m}$. The CRL was located $28.5 \mathrm{~m}$ from the undulator source and upstream of the monochromator. The pre-focusing CRL focused $12.7 \mathrm{keV}$ $\mathrm{X}$-rays to a distance of $35.5 \mathrm{~m}$ downstream, thus increasing the photon flux at the sample position.

The sample environment was represented with several micro-manipulators that could carry pinholes or test objects for imaging and microscopy. The manipulators had three orthogonal translations for the perfect sample alignment and were located upstream of the objective nano-goniometer.

As an objective lens, we used a polymer compound refractive lens ( $\mathrm{PCRL}_{30}$, Fig. 1), comprised of 30 individual double-concave parabolic polymer lenses, described by Petrov

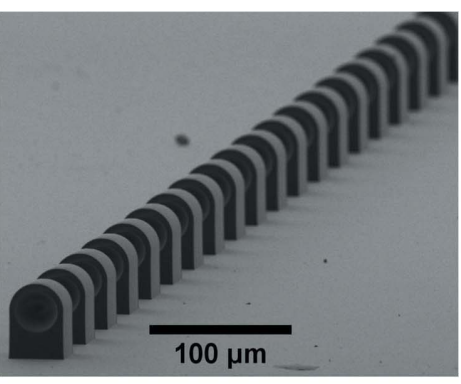

(a)

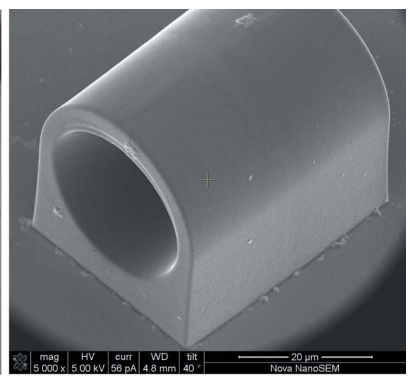

(b)
Figure 1

Scanning electron micrograph of $(a)$ the $\mathrm{PCRL}_{30}$ and $(b)$ one individual lens.

et al. (2017). The printing parameters were $13 \mathrm{~mW}$ and $400 \mathrm{~m} \mathrm{~s}^{-1}$. The polymer material had the formula $\mathrm{C}_{21} \mathrm{H}_{36} \mathrm{O}_{8} \mathrm{SiS}$ and a density of $1.2 \mathrm{~g} \mathrm{~cm}^{-3}$. Each single lens was expected to have a radius of curvature, $R$, of $5 \mu \mathrm{m}$ and a physical aperture, $A$, of $24 \mu \mathrm{m}$. According to the calculations, the focal distance and the diffraction-limited resolution (Schroer et al., 2013) of the $\mathrm{PCRL}_{30}$ at $12.7 \mathrm{keV}$ X-ray energy were expected to be $F=5 \mathrm{~cm}$ and $S=70 \mathrm{~nm}$, respectively. $\mathrm{PCRL}_{30}$ was installed on the six-axis nano-goniometer allowing for all necessary translations and rotations with nanometre accuracy.

For image acquisition, we used the PCO-2000 CCD camera coupled with a monochromatic beam optical system (Optique Peter) and a 20-fold objective. It gave an effective pixel size of $0.37 \mu \mathrm{m}$ within the 2048 pixels $\times 2048$ pixels field of view. The detector was mounted downstream of the $\mathrm{PCRL}_{30}$ objective at a separate bench with all necessary translations, allowing us to vary the $\mathrm{PCRL}_{30}$-to-detector distance from several millimetres to $3 \mathrm{~m}$.

\section{X-ray focusing}

First of all, we tested PCRL $\mathrm{P}_{30}$ in focusing mode (Fig. 2) to check its optical properties such as focal length, spot size and absence (or presence) of aberrations. Knowing that the ideal $\mathrm{PCRL}_{30}$ would have a focal distance of $5 \mathrm{~cm}$, we scanned the space near this position along the lens optical axis, recording images of the focal spot with the detector. A strong astigmatism was discovered, which manifested itself in the rays propagating in two perpendicular planes and having different foci (Hecht, 2002). Thus, vertical and horizontal lines of the object were in sharp focus at two different distances: $F_{\mathrm{v}}=$ $37 \mathrm{~mm}$ and $F_{\mathrm{h}}=45 \mathrm{~mm}$, respectively [Figs. 3( $\left.a\right)$ and 3(b)]. At

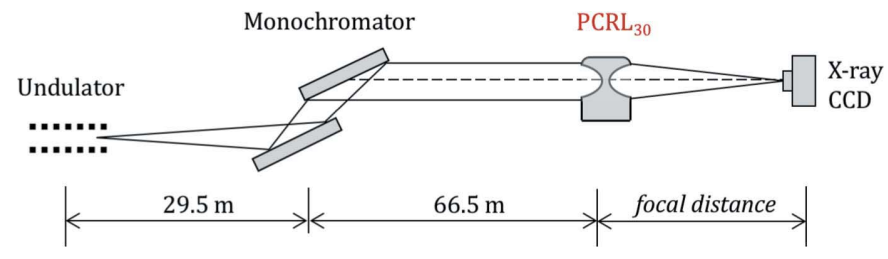

Figure 2

Layout of the focusing experiment. 


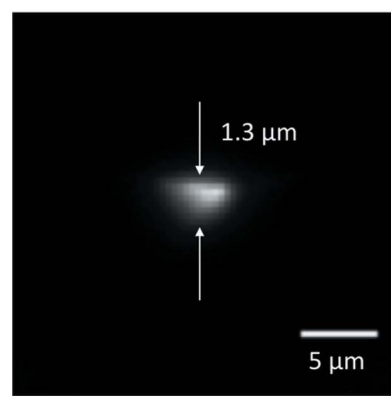

(a)

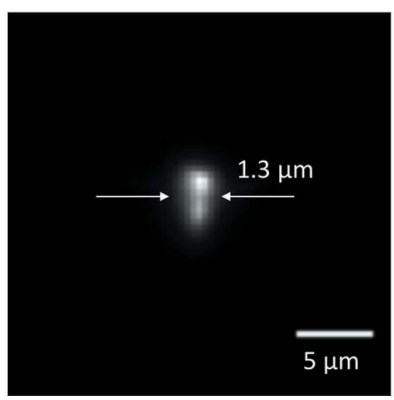

(b)

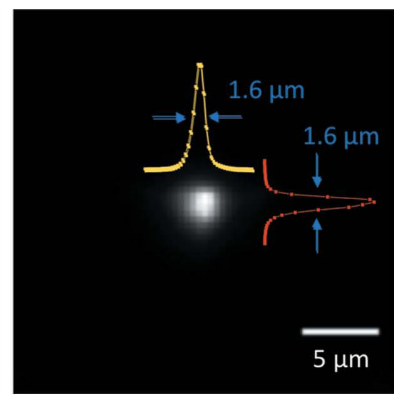

(c)
Figure 3

X-ray images of lens sharp foci in the $(a)$ vertical $\left(F_{\mathrm{v}}=37 \mathrm{~mm}\right)$ and $(b)$ horizontal $\left(F_{\mathrm{h}}=45 \mathrm{~mm}\right)$ directions. $(c)$ The circle of least confusion is located in between at $F_{1}=41 \mathrm{~mm}$.

The presence of astigmatism can account for the manufacturing procedure. During the two-photon polymerization, a minimal polymerizable volume (voxel) is an ellipsoid of revolution (spheroid), vertically elongated along the optical axis of the laser beam. The elongation depends on the laser irradiation power and becomes more pronounced with its increase (Sakellari et al., 2012). The further lens shape is an envelope of elementary spheroids with the centers lying on a given parabolic trajectory [Fig. 4(a)]. Estimating

these distances, the spots had an equal minimum size of $1.3 \mu \mathrm{m}$ (full width at half-maximum; FWHM), which is significantly larger than the diffraction limit $S$ of $70 \mathrm{~nm}$ even if one takes into account the finite camera pixel of $0.37 \mu \mathrm{m}$.

Between $F_{\mathrm{v}}$ and $F_{\mathrm{h}}$, we found the circle of least confusion [Fig. 3(c)]. It was located at a distance of $F_{1}=41 \mathrm{~mm}$, where $\mathrm{PCRL}_{30}$ focused the radiation symmetrically into a $1.6 \mu \mathrm{m} \times$ $1.6 \mu \mathrm{m}$ (FWHM) spot. Yet the experimentally determined value $F_{1}$ differs from the expected value of $F=50 \mathrm{~mm}$, indicating that each single biconcave lens has an average curvature radius $R=4 \mu \mathrm{m}$ that is smaller than designed value of $5 \mu \mathrm{m}$.

The astigmatism can be described with the relative parameter $\Delta$, which shows an offset of both foci and lens curvature radii from their average values,

$$
\Delta=\left|\frac{F_{\mathrm{v}}-F_{\mathrm{h}}}{F_{\mathrm{v}}+F_{\mathrm{h}}}\right| \times 100 \%=\left|\frac{R_{\mathrm{v}}-R_{\mathrm{h}}}{R_{\mathrm{v}}+R_{\mathrm{h}}}\right| \times 100 \% .
$$

In the present case, $\Delta_{1}=10 \%$, meaning that lens radii in the vertical and horizontal directions are different and equal to $R_{\mathrm{v}}=3.6 \mu \mathrm{m}$ and $R_{\mathrm{h}}=4.4 \mu \mathrm{m}$, respectively. the voxel shape to be a circle (with radius $a=b=0.3 \mu \mathrm{m}$ ) in the horizontal plane [Fig. 4(b)] and a vertically elongated ellipse ( $a=0.5 \mu \mathrm{m}$ and $b=0.3 \mu \mathrm{m})$ in the vertical plane, the shape of the envelope $y$ may be calculated using the following system of equations for a one-parameter family of plane curves,

$$
\begin{aligned}
f(x, y, t) & =0, \\
f^{\prime}(x, y, t) & =0 .
\end{aligned}
$$

For our specific cases of an ellipse and a circle, this system transforms to

$$
\begin{gathered}
\frac{(x-t)^{2}}{a^{2}}+\frac{\left(y-t^{2} / 2 R_{0}\right)^{2}}{b^{2}}=1, \\
\frac{(x-t)}{a^{2}}+\frac{t}{R_{0}} \frac{\left(y-t^{2} / 2 R_{0}\right)}{b^{2}}=0,
\end{gathered}
$$

where $R_{0}=5 \mu \mathrm{m}$ is the designed lens parabolic parameter (radius); $\left(t, t^{2} / 2 R_{0}\right)$ and $(x, y)$ are the plane coordinates of the voxel center and envelope, respectively [Fig. 4(a)]. After solving equation (3), we found envelope shapes for both the vertical $\left[y_{\mathrm{v}}(x)\right]$ and horizontal $\left[y_{\mathrm{h}}(x)\right]$ planes. If every small portion of each envelope is then approximated by its own tangential parabola with curvature radii of $R_{\mathrm{h}, \mathrm{v}}(x)=$ $1 / y_{\mathrm{h}, \mathrm{v}}{ }^{\prime \prime}(x)$, the radii will take the values that are shown in Fig. 4(c). These numbers mean that the lens shape strongly deviates from the ideal one in the center, while becoming closer to the designed one towards the edges.

During the subsequent developing step (dissolving of unpolymerized resin), the volume of the polymer material further shrinks due to solvent evaporation. Moreover, the shrinkage of the lens is not isotropic - its bottom is attached to the substrate and cannot change dimensions similarly to the upper part. Altogether, the above leads to the reduction of the lens radii, spherical aberrations and to astigmatism which we have experimentally observed.

Schematic representation of $(a)$ how the resulting lens envelope deviates from the initial laser trajectory due to $(b)$ voxel ellipticity in the vertical plane. (c) Deviation of radii in the vertical and horizontal lens planes from their designed value of $5 \mu \mathrm{m}$. 


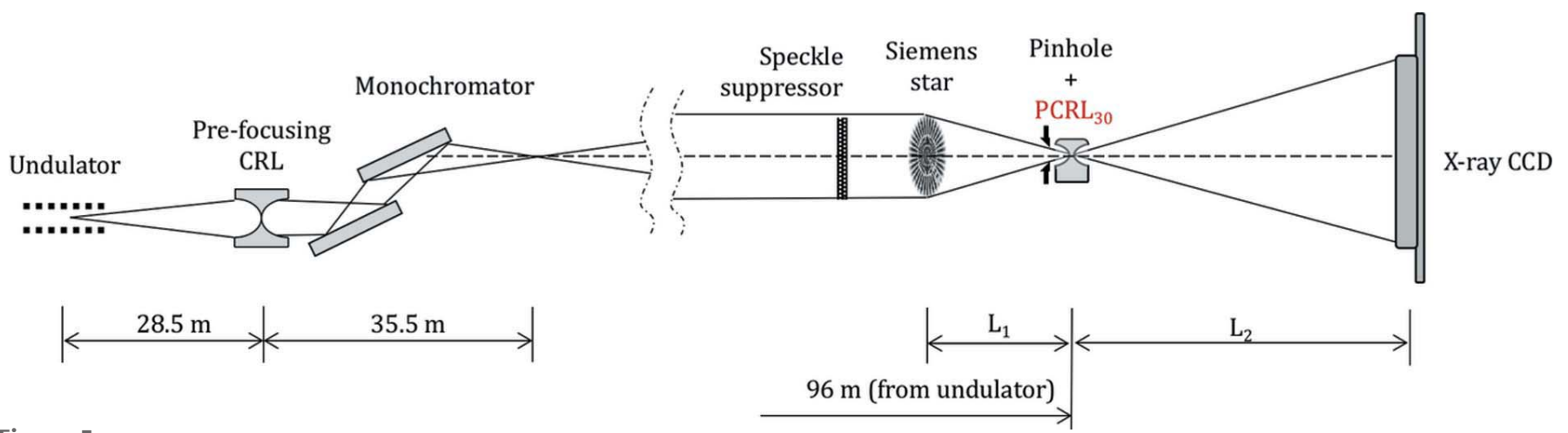

Figure 5

Layout of the experiment in X-ray microscopy mode. Note that parts of the drawing before and after the gap have different scales.

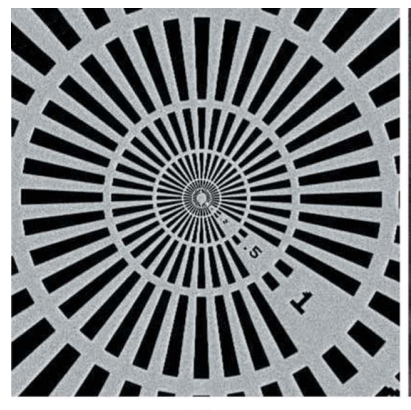

(a)

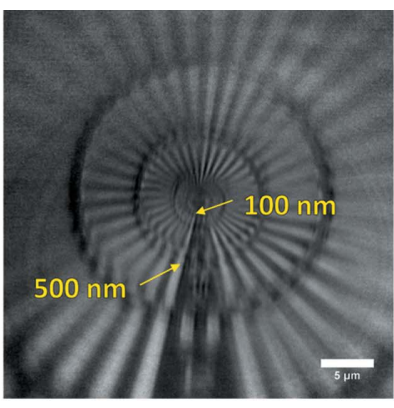

(b)

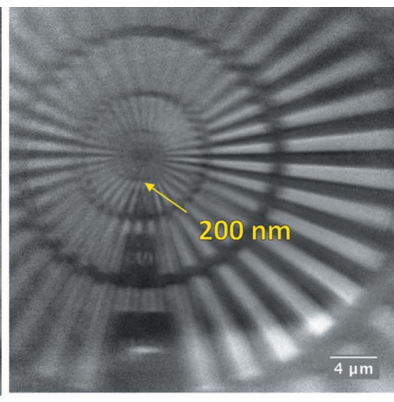

(c)

Figure 6

(a) Scanning electron and (b) flat-field-corrected X-ray images of the Siemens star. (c) The resolution remains the same after $5.5 \mathrm{~h} \mathrm{X}$-ray exposure.

\section{Full-field X-ray microscopy}

PCRL $_{30}$ was also tested in full-field X-ray microscopy mode (Fig. 5) to refine the maximum resolution of the lens and the image quality. In this mode, we used the pre-focusing CRL located at the optics hutch. A Siemens star and a $25 \mu \mathrm{m}$ pinhole were also mounted upstream of the PCRL $\mathrm{PL}_{30}$ on the micro-manipulators. A Siemens star is a test object [Fig. 6(a)] that consists of a pattern of 'spokes' etched in SiN and covered by a $500 \mathrm{~nm}$ layer of Ta (XRESO-50HC, NTT-AT, Japan). The Siemens star was located at a distance $L_{1}$ of $43 \mathrm{~mm}$ from the PCRL $_{30}$. According to the thin lens formula

$$
\frac{1}{F}=\frac{1}{L_{1}}+\frac{1}{L_{2}}
$$

and assuming that the focal distance $F$ is equal to $F_{1}=41 \mathrm{~mm}$ (as from the previous paragraph), we located the X-ray detector at $L_{2}=885 \mathrm{~mm}$ from the PCRL 30 . Thus, we achieved a lens magnification of $L_{2} / L_{1}=20.6$, giving an effective detector pixel size of $18 \mathrm{~nm}$.

To ensure the best image quality, we used a speckle suppressor (Goikhman et al., 2015) - a rotating plate of porous nanoberyllium that homogeneously scatters X-rays upstream of the sample. To improve the contrast and signal-to-noise ratio, we also applied a flat-field correction [Fig. 6(b)], dividing the image with the mounted Siemens star by the image with the unmounted one. It allowed us to resolve the $100 \mathrm{~nm}$ and $200 \mathrm{~nm}$ bars in the vertical and horizontal directions, respecwith a flux density of $7 \times 10^{11}$ photons s${ }^{-1} \mathrm{~mm}^{-2}$. The procedure lasted for $5.5 \mathrm{~h}$. During that time, individual lenses of the PCRL 30 absorbed an average dose of $\sim 15 \times 10^{6} \mathrm{~Gy}$ each. X-ray radiograms of the $\mathrm{PCRL}_{30}$ before and after exposure (Fig. 7) demonstrate non-uniform polymer lens shrinkage; lenses were almost unchanged around the substrate-lens conjunction because of the mechanical stress and therefore higher stability. However, they shrunk significantly around the freestanding top. After the exposure, we roughly estimated the decrease in size to be $17 \pm 2 \%$ on average, irrespective of the complexity of the lens shape.

We also repeated the focusing experiment to see the change in the focal distance. The PCRL 30 focused the radiation in the

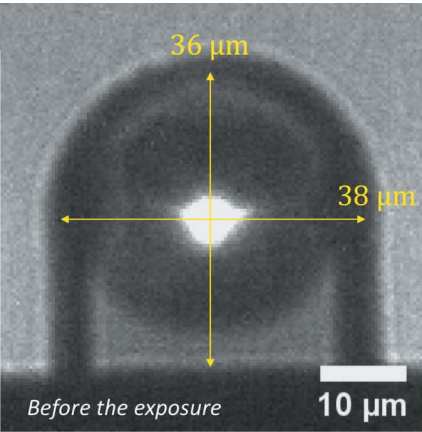

(a)

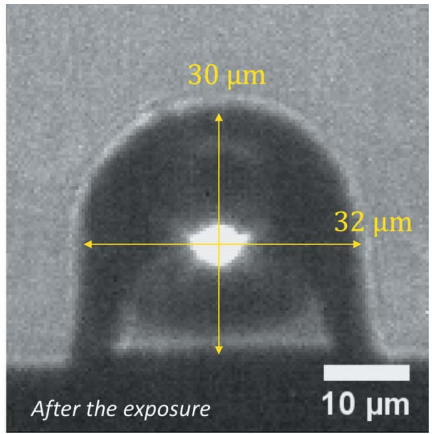

(b)
Figure 7

X-ray radiograms of the $\mathrm{PCRL}_{30}$ were taken under the same experimental conditions $(a)$ before and $(b)$ after $5.5 \mathrm{~h} \mathrm{X-ray} \mathrm{exposure.}$ 
circle of least confusion with $1.8 \mu \mathrm{m} \times 1.8 \mu \mathrm{m}$ size and at a focal distance $F_{2}=35 \mathrm{~mm}$. The distance $F_{2}$ is $15 \%$ smaller than $F_{1}$, which corresponds to our observations of geometrical size reduction. The $\mathrm{PCRL}_{30}$ still had astigmatism, with sharp vertical and horizontal foci at $F_{\mathrm{v} 2}=32 \mathrm{~mm}$ and $F_{\mathrm{h} 2}=38 \mathrm{~mm}$, respectively. Estimating the astigmatism again with the parameter $\Delta$, we obtain a value $\Delta_{2}=8.5 \%$.

In subsequent experiments, we studied whether the degraded $\mathrm{PCRL}_{30}$ preserved the imaging qualities. We repeated the X-ray microscopy experiment with the corrected distances $L_{1}=36.5 \mathrm{~mm}$ and $L_{2}=851 \mathrm{~mm}$ [as in equation (4)]. As a result, we obtained a 23 -fold magnified X-ray image [Fig. 6(c)]. The image was almost identical to the one obtained before the radiation damage $-200 \mathrm{~nm}$ bars were the last ones clearly visible and the lenses were still successfully working as microscopic objective lenses, even though their radii were non-uniformly reduced.

The experiment has revealed that X-ray irradiation causes a continuous degradation of polymer material, namely polymer chain scission or crosslinking. A detailed description of the lens degradation mechanism requires further research and is beyond the scope of this paper. From a practical point of view, one could only state that, although lenses suffer from continuous degradation-induced shrinkage, there are no volume cracks, voids or pores, because those would be immediately seen (by having a strong effect) on the X-ray micrographs.

\section{Conclusions}

Our experiments showed that the polymer lenses worked successfully, achieving a resolution of 100-200 $\mathrm{nm}$ in full-field imaging mode, which was relatively close to their diffraction limit of $70 \mathrm{~nm}$ using the current setup. Nevertheless, lenses had strong astigmatism arising from the manufacturing process, which led to image distortions in microscopy mode. It also reduced their focusing performance, not allowing one to achieve the expected nanometre size of the focal spot.

One could make several changes to improve the lens geometry, radiation stability and overall quality. First of all, the voxel ellipticity could be reduced by decreasing the average dose delivered by the laser in two-photon polymerization. Second, knowing the voxel size and shape, we could introduce the corrected laser trajectory in order to achieve a parabolic envelope as a result. Then, there are several ways to deal with polymerization shrinkage as it is a well known problem in two-photon lithography. One could use special polymer materials with significantly reduced shrinkage (Ovsianikov et al., 2009). However, we should pay special attention to the chemical composition of a polymer, because the presence of heavy elements in the CRL will lead to increased X-ray absorption. Another approach could be used to compensate for the shrinkage on the stage of lens design (Sun et al., 2004) - to reduce the mechanical stress in the substrate-lens conjunction with the use of sponge-like or anchor-like supporting structures (Maruo et al., 2009). This will lead to a more uniformed shrinkage (including that induced by X-ray exposure), thus reducing the astigmatism. Finally, we may improve the mechanical and X-ray radiation resistance of the lens, applying post UV-curing methods with an increased degree of crosslinking while preserving the same resolution of 3D printing (Oakdale et al., 2016).

The improvements proposed above could help to enhance the lens quality, allowing sufficient resolution for nanofocusing applications to be achieved. To summarize, even though the polymer refractive lenses still require some technological improvement, they are already promising as versatile compact devices for X-ray microscopy. Due to their short focal distance and in contrast to beryllium CRLs, they can be easily integrated into most optical schemes. Besides synchrotrons, polymer lenses could also be extremely useful at laboratory X-ray sources where compactness affects the recorded X-ray intensity.

\section{Acknowledgements}

We thank A. Petrov (MSU) and ESRF ID13 staff for their help in this research.

\section{Funding information}

The research was supported by The Russian Ministry of Education and Science (grant No. 14.Y26.31.0002, 14.W03.31.0008 - lens printing and tests), the Russian Science Foundation (15-12-00065 - scanning electron microscopy), the Russian Academic Excellence Project at IKBFU and by the Quantum technology centre at Moscow State University. VY was supported by the State Task No. 075-00475-19-00.

\section{References}

Aristov, V., Grigoriev, M., Kuznetsov, S., Shabelnikov, L., Yunkin, V., Weitkamp, T., Rau, C., Snigireva, I., Snigirev, A., Hoffmann, M. \& Voges, E. (2000). Appl. Phys. Lett. 77, 4058-4060.

Aristov, V. V., Grigoriev, M., Kuznetsov, S., Shabelnikov, L., Yunkin, V., Rau, C., Snigirev, A., Snigireva, I., Weitkamp, T., Hoffmann, M. \& Voges, E. I. (2001). Proc. SPIE, 4145, 285-293.

Dimper, R., Reichert, H., Raimondi, P., Ortiz, L. S., Sette, F. \& Susini, J. (2014). ESRF Upgrade Programme Phase II (2015-2022) Technical Design Study, http://www.esrf.fr/Apache_files/Upgrade/ ESRF-orange-book.pdf.

Goikhman, A., Lyatun, I., Ershov, P., Snigireva, I., Wojda, P., Gorlevsky, V., Semenov, A., Sheverdyaev, M., Koletskiy, V. \& Snigirev, A. (2015). J. Synchrotron Rad. 22, 796-800.

Hecht, E. (2002). Optics, 4th ed. New York: Addison-Wesley.

Lengeler, B. (2004). AIP Conf. Proc. 705, 748-751.

Lengeler, B., Schroer, C., Tümmler, J., Benner, B., Richwin, M., Snigirev, A., Snigireva, I. \& Drakopoulos, M. (1999). J. Synchrotron Rad. 6, 1153-1167.

Lengeler, B., Schroer, C. G., Kuhlmann, M., Benner, B., Günzler, F. T., Kurapova, O., Zontone, F., Snigirev, A. \& Snigireva, I. (2005). J. Phys. D Appl. Phys. 38, A218-A222.

Marchesini, S., He, H., Chapman, N., Hau-Riege, P., Noy, A., Howells, R., Weierstall, U. \& Spence, H. (2003). Phys. Rev. B, 68, 140101.

Maruo, S., Hasegawa, T. \& Yoshimura, N. (2009). Opt. Express, 17, 20945-20951.

Mirzaeimoghri, M., Morales Martinez, A., Panna, A., Bennett, E. E., Lucotte, B. M., DeVoe, D. L. \& Wen, H. (2018). PLoS One, 13, e0203319.

Oakdale, J. S., Ye, J., Smith, W. L. \& Biener, J. (2016). Opt. Express, 24, 27077-27086. 
Ovsianikov, A., Shizhou, X., Farsari, M., Vamvakaki, M., Fotakis, C. \& Chichkov, B. N. (2009). Opt. Express, 17, 2143-2148.

Petrov, A. K., Bessonov, V. O., Abrashitova, K. A., Kokareva, N. G., Safronov, K. R., Barannikov, A. A., Ershov, P. A., Klimova, N. B., Lyatun, I. I., Yunkin, V. A., Polikarpov, M., Snigireva, I., Fedyanin, A. A. \& Snigirev, A. (2017). Opt. Express, 25, 14173-14181.

Polikarpov, M., Polikarpov, V., Snigireva, I. \& Snigirev, A. (2016). Phys. Procedia, 84, 213-220.

Sakellari, I., Kabouraki, E., Gray, D., Purlys, V., Fotakis, C., Pikulin, A., Bityurin, N., Vamvakaki, M. \& Farsari, M. (2012). ACS Nano, 6 , 2302-2311.

Sanli, U. T., Ceylan, H., Bykova, I., Weigand, M., Sitti, M., Schütz, G. \& Keskinbora, K. (2018). Adv. Mater. 30, 1802503.

Schroer, C. G., Brack, F.-E., Brendler, R., Hönig, S., Hoppe, R., Patommel, J., Ritter, S., Scholz, M., Schropp, A., Seiboth, F.,
Nilsson, D., Rahomäki, J., Uhlén, F., Vogt, U., Reinhardt, J. \& Falkenberg, G. (2013). Proc. SPIE, 8848, 884807.

Schroer, C. G., Kurapova, O., Patommel, J., Boye, P., Feldkamp, J., Lengeler, B., Burghammer, M., Riekel, C., Vincze, L., Van Der Hart, A. \& Küchler, M. (2005). Appl. Phys. Lett. 87, 1-3.

Snigirev, A., Hustache, R., Duboc, P., Massonnat, J.-Y., Claustre, L., Van Vaerenbergh, P., Snigireva, I., Grigoriev, M. \& Yunkin, V. (2007). Proc. SPIE, 6705, 670511.

Snigirev, A., Kohn, V., Snigireva, I. \& Lengeler, B. (1996). Nature, 384, 49-51.

Snigirev, A. \& Snigireva, I. (2008). C. R. Phys. 9, 507-516.

Snigireva, I., Vaughan, G. B. M., Snigirev, A., McNulty, I., Eyberger, C. \& Lai, B. (2011). AIP Conf. Proc. 1365, 188-191.

Sun, H. B., Suwa, T., Takada, K., Zaccaria, R. P., Kim, M. S., Lee, K. S. \& Kawata, S. (2004). Appl. Phys. Lett. 85, 3708-3710. 